\title{
Jean Siraudeau (Cholet, 6 juin 1922 - Bouchemaine, 9 avril 2021), archéologue angevin
}

Gérard Aubin, François Comte, Maxime Mortreau et Daniel Prigent

\section{OpenEdition}

Édition électronique

URL : https://journals.openedition.org/rao/7557

DOI : $10.4000 /$ rao. 7557

ISSN : 1775-3732

Éditeur

Presses universitaires de Rennes

\section{Édition imprimée}

Date de publication : 21 décembre 2021

Pagination : 363-365

ISBN : 978-2-7535-8668-0

ISSN : 0767-709X

\section{Référence électronique}

Gérard Aubin, François Comte, Maxime Mortreau et Daniel Prigent, « Jean Siraudeau (Cholet, 6 juin 1922 - Bouchemaine, 9 avril 2021), archéologue angevin », Revue archéologique de l'Ouest [En ligne], 37 | 2021, mis en ligne le 21 décembre 2021, consulté le 10 octobre 2022. URL : http:// journals.openedition.org/rao/7557 ; DOI : https://doi.org/10.4000/rao.7557 


\section{Jean Siraudeau}

\section{(Cholet, 6 juin 1922 - Bouchemaine, 9 avril 2021), archéologue angevin}

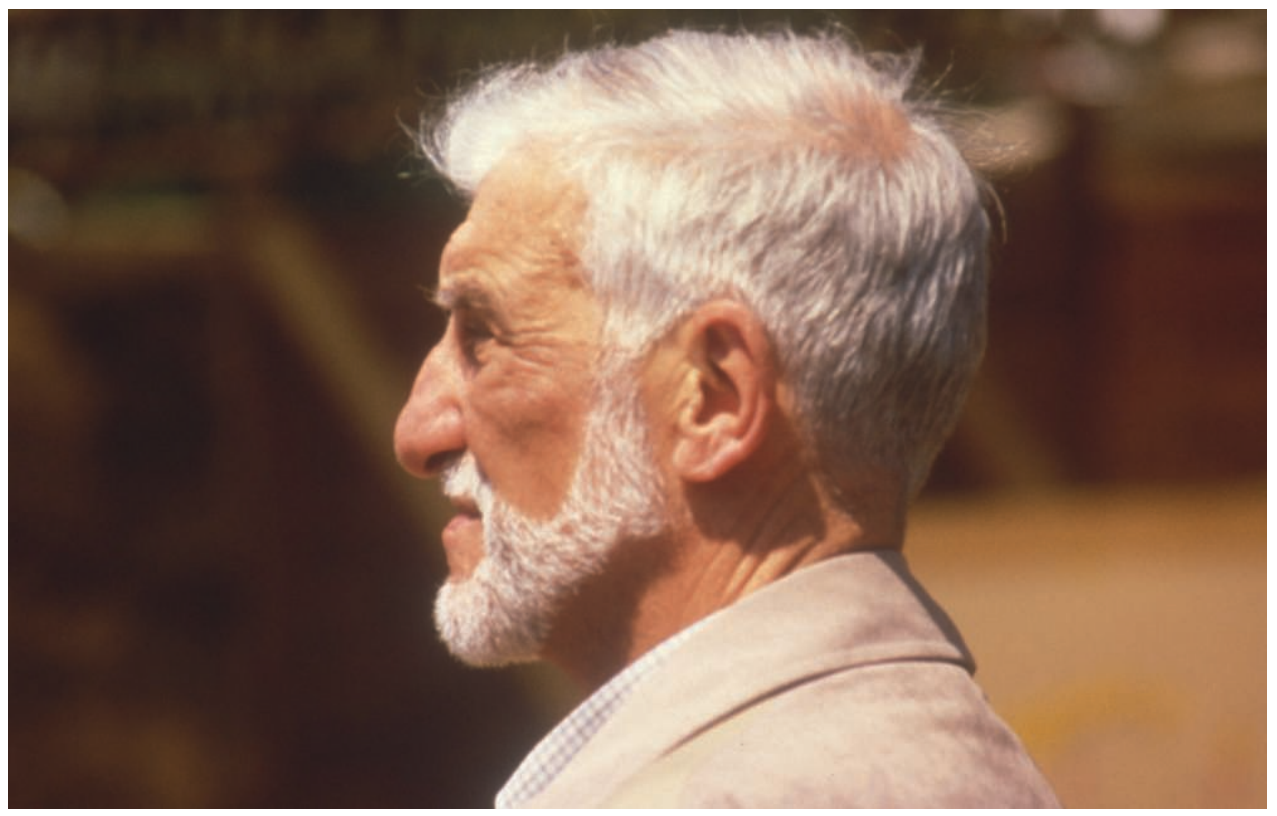

Jean Siraudeau

(cliché Jean Rioufreyt).

L'annonce du décès, dans sa quatre-vingt-dix-neuvième année, de Jean Siraudeau, a suscité une sincère émotion dans notre communauté. Les réactions de plusieurs générations d'archéologues ont été unanimes à souligner sa passion pour le patrimoine angevin, son dévouement et sa disponibilité, son exigence de précision et son souci de transmission. Cet hommage à l'un des bâtisseurs de l'archéologie d'aujourd'hui évoque brièvement quelques facettes de son parcours.

\section{L'ARCHÉOLOGUE DE TERRAIN}

Issu d'une famille originaire des Mauges, Jean Siraudeau a cependant effectué l'essentiel de ses études et de sa carrière professionnelle à Angers. Élève de l'Institution Mongazon puis du lycée David-d'Angers où il passa le baccalauréat en 1941, il commença son droit à l'université catholique de l'Ouest, vite interrompu par la guerre. Ayant réussi à échapper au STO, il entra dans l'imprimerie familiale de la place de la Visitation et en fut l'un des responsables commerciaux jusqu'à sa retraite en 1984.
L'archéologie devint une passion sur le tard après sa rencontre vers 1961 avec le Dr Michel Gruet, géologue, conservateur-adjoint du muséum d'Angers et correspondant départemental des Antiquités préhistoriques.

C'est comme bénévole le dimanche et les vacances d'été sur les chantiers de fouilles préhistoriques du $\mathrm{D}^{\mathrm{r}}$ Gruet que J. Siraudeau s'initia à l'archéologie. Chargé d'étudier les vestiges antiques trouvés dans les dolmens, il approfondit ses connaissances sur cette période auprès de René Sanquer en participant à ses chantiers dans le Finistère et plus tard, auprès de Fanette Laubenheimer, à Sallèles-d'Aude.

Son premier grand chantier archéologique en tant que responsable fut celui - mémorable - de la place du Ralliement en 1971, acte fondateur de l'archéologie urbaine à Angers. Dès 1965, il était toutefois conscient de la destruction des sols en dénonçant l'absence du respect de la législation archéologique et l'impéritie des entrepreneurs et élus locaux. La décennie 1970 fut ainsi une période d'intense activité archéologique de terrain, comme en témoigne la rédaction de dix-huit rapports de sondages ou de ce qu'on appelait alors des « fouilles 
de sauvetage urgent ". Sans grands moyens humains et financiers, il forma néanmoins une petite poignée de bénévoles qui s'attachèrent à suivre les travaux de voirie (rues Saint-Julien, du Vollier...), à réaliser des interventions d'environ deux semaines, faute de mieux (avenue de la Blancheraie, mail de la Préfecture...) jusqu’à des travaux archéologiques de plus grande ampleur (rue Delaâge, Bibliothèque municipale, Maison du Tourisme...). Les résultats obtenus furent essentiels pour la connaissance de l'évolution d'Angers antique, la ville médiévale restant encore inconnue car assez éloignée des préoccupations de beaucoup de chercheurs de l'époque.

Si J. Siraudeau est surtout intervenu sur la ville d'Angers, on ne peut négliger d'autres opérations d'ampleur très variée dans le département de Maine-et-Loire, réalisées à la demande de ce qui était alors la direction régionale des Antiquités historiques. Ainsi, en 1975, il fit classer en urgence le site du théâtre antique de Sainte Gemmessur-Loire, menacé de destruction. Le Moyen Âge, qui n'était pas sa période de prédilection, a toutefois donné lieu à plusieurs interventions. Nous citerons simplement la petite nécropole mérovingienne de Galmer à Méon, celle de Thouarcé où s'étend vraisemblablement l'une des plus grandes nécropoles angevines en dehors de la cité et la première fouille d'urgence dans l'abbaye de Fontevraud. À la suite de la découverte fortuite de sarcophages près de l'église de Brain-sur-l'Authion, dont la paroisse est antérieure à l'an mil, la fouille de la chapelle orientée a livré la base de l'élévation vraisemblablement romane de celle-ci.

La création du service archéologique départemental de Maine-et-Loire en 1979, puis du service municipal d'Angers en 1982, consécutive à la fouille menée en urgence à la suite de la découverte et de la destruction de thermes antiques, l'essor des services régionaux d'archéologie, la mise en place de l'Afan puis de l'Inrap, allaient conduire au développement de nouvelles procédures, notamment à l'instauration de campagnes de fouilles continues. La fouille de l'îlot 25 (rue Talot/rue Delaâge) à Angers constituera sa dernière activité de terrain en tant que responsable d'opération, ce qui ne l'empêchera pas d'apporter ultérieurement son soutien lors d'interventions diverses, notamment celles de Michel Gruet. Dès lors, son activité se concentrera sur l'analyse des mobiliers antiques mis au jour lors d'opérations menées par différents intervenants angevins, et ce jusqu'à ces derniers mois. Cette période de réflexion lui a aussi permis la rédaction d'une fort utile synthèse sur les amphores, parue en 1988, de même que l'élaboration de plusieurs parties d'un des premiers Document d'évaluation du patrimoine archéologique des villes de France (1990).

François Comte et Daniel Prigent

\section{LE CÉRAMOLOGUE}

Jean Siraudeau a mesuré très tôt l'apport de la céramologie à la datation des contextes stratigraphiques fouillés. En 1976, il présentait une communication inédite au congrès de Saintes de la jeune SFECAG (Société française d'étude de la céramique antique en Gaule), sur les ateliers de potiers fouillés en 1971, place du Ralliement, et en 1974 au 14 rue Delaâge. Dans les années 1980, il intégrait le Groupe de recherches sur les amphores en Gaule romaine, dirigé alors par Fanette Laubenheimer, et en devenait l'un des membres actifs.

Son ouvre majeure restera Amphores romaines des sites angevins et leur contexte archéologique. Cet ouvrage, préparé avec l'aide de la sous-direction de l'archéologie et édité à compte d'auteur avec le concours des collectivités en 1988, s'inscrivait dans le «Corpus des amphores découvertes dans l'Ouest de la France " initié par René Sanquer, et faisait suite à un premier volume consacré en 1982 par Patrick Galliou aux amphores tardo-républicaines de Bretagne et des Pays de la Loire. Il s'agissait de réaliser une synthèse à partir de l'ensemble des collections issues des fouilles menées à Angers depuis la naissance de cette discipline. Avec la collaboration des responsables de fouilles, il effectua un inventaire minutieux et quasiexhaustif de tous les tessons d'amphores découverts et rédigea d'utiles notices synthétisant les informations de ces différents chantiers. Pour un futur nouveau tome de cette collection, l'infatigable chercheur voulait explorer la piste des analyses physico-chimiques pour vérifier la pertinence des identifications qu'il avait pu faire pour le matériel d'Angers. À cette fin, il sollicita des crédits auprès de plusieurs partenaires dont la DRAC pour lancer dès 1990 un ambitieux programme d'analyses confié au laboratoire de céramologie de Lyon dirigé par Maurice Picon. Les résultats de ces travaux sont malheureusement restés largement confidentiels.

Il entreprit aussi l'inventaire des amphores conservées dans les dépôts archéologiques des Pays de la Loire. Très attaché à sa ville natale, il réalisa bénévolement, en 1992, le classement, l'inventaire et les dessins de la céramique gallo-romaine conservée au musée d'Art et d'Histoire de Cholet. Il participa à plusieurs projets de publication dont celui des fouilles du château d'Angers. Son ultime regret aura été de ne pas voir la publication du site des Pichelots aux Alleuds que son ami le regretté $\mathrm{D}^{\mathrm{r}}$ Michel Gruet avait fouillé et dont il avait étudié les amphores.

Maxime Mortreau 


\section{LE CORRESPONDANT DU DIRECTEUR DES ANTIQUITÉS}

Jean Siraudeau a été mon correspondant en Maine-etLoire de 1978 à 1989. Cette fonction très officielle, créée par décret en 1945, visait à pallier l'absence de personnel dans les directions des Antiquités. Le rôle du correspondant, défini par un arrêté en 1969, consistait, moyennant une très modeste indemnité, à tenir le directeur informé du déroulement des chantiers en cours, des découvertes fortuites, des fouilles clandestines, des projets de travaux menaçant les sites, voire à le représenter. En outre, au gré des rencontres organisées avec ses collègues des autres départements, il contribuait à la réflexion régionale sur la pratique et l'évolution de la discipline.

De Jean Siraudeau, je conserve l'image d'un interlocuteur compétent, actif et bienveillant, dont la fidélité n’a jamais été prise en défaut. Engagé dans les sauvetages - son nom restera associé à ceux des chantiers angevins de la rue Delaâge, de la Bibliothèque Toussaint, de la rue Kellermann, de la Maison du Tourisme, pour ne citer que les plus importants -, il accompagna l'émergence d'une démarche d'archéologie préventive et accueillit chaleureusement la création d'un poste d'archéologue municipal par la ville d'Angers (1982) comme il avait applaudi à celle d'archéologue départemental (1979). À dire vrai, son influence ne fut pas étrangère à ces innovations pionnières dans les Pays de la Loire. Il put alors réorienter l'essentiel de son activité vers la réalisation avec F. Comte d'un Plan archéologique urbain et l'étude du mobilier. Sa connaissance de la ville mais aussi du milieu angevin m'a été particulièrement précieuse pour administrer un territoire réputé difficile et coordonner l'activité de passionnés d'archéologie parfois turbulents; lors de nos entretiens téléphoniques, généralement hebdomadaires, il me faisait part des problèmes en cours et souvent me proposait en même temps des solutions. Nos tournées sur le terrain, parfois en compagnie du $\mathrm{D}^{\mathrm{r}}$ Gruet - correspondant de mon collègue préhistorien - étaient toujours très fructueuses et empreintes de convivialité.

Je n'aurais garde d'oublier la Revue archéologique de l'Ouest dont, en tant qu'imprimeur cette fois, il facilita le lancement en nous prodiguant ses conseils. De 1984 à la fin des années 1990, c'est l'imprimerie Siraudeau qui se chargea de la fabrication de la revue et de ses suppléments.

\section{Bibliographie sélective}

Siraudeau J., 1972, [1978], "L'époque gallo-romaine », dans Lebrun F. (dir.), Histoire des Pays de la Loire (Orléanais, Touraine, Anjou, Maine), Toulouse, Privat, p. 55-80 [réédit. Genève, Famot, p. 59-83].

Siraudeau J., 1982, "Angers ", Archéologie urbaine. Actes du Colloque international (Tours, 17-20 novembre 1980), Paris, Afan, p. 295-299.

Siraudeau J., 1988, Amphores romaines des sites angevins et leur contexte archéologique, Bouchemaine, J. Siraudeau, 238 p. (Corpus des amphores découvertes dans l'Ouest de la France, 2).

Siraudeau J., 1989, "Amphores d'importation et d'origine gauloise à Angers ", Amphores romaines et histoire économique. Dix ans de recherche. Actes du colloque international de Sienne (22-24 mai 1986), Rome, École française de Rome, p. 654-655 (Publications de l'École française de Rome, 114).

Comte F. et Siraudeau J., 1990, Angers. Document d'évaluation du patrimoine archéologique urbain, Paris, Association pour les fouilles archéologiques nationales et Tours, Centre national d'archéologie urbaine, 129 p., 13 pl. h.-t. (Documents d'évaluation du patrimoine archéologique des villes de France).

Siraudeau J., 1992, Inventaire de la céramique gallo-romaine conservée au musée des Arts de Cholet, tapuscrit, 34 p. et $33 \mathrm{pl}$.

Siraudeau J., 1999, [textes et notices de catalogue], dans Santrot M.-H., Santrot J. et Meuret J.-C. (dir.), Nos ancêtres les Gaulois aux marges de l'Armorique. Catalogue de l'exposition du musée Dobrée, Nantes, Conseil général de Loire-Atlantique, $2^{\text {e }}$ éd. : p. 24 ("Le village gaulois des Pichelots (Les Alleuds, Maine-et-Loire)»), p. 25 ("Cuvelage d'un puits à section carrée »), p. 28 ("Dormants de meules à grain (metae)»), p. 34 ("Pesons ou lests de métier à tisser »), p. 49 ("Céramiques des Pichelots "), p. 52-53 (" Production des potiers de l'atelier de la rue Delaâge, à Angers »), p. 92-93 («Du vin d'Italie par l'Atlantique et la Loire ").

De 1971 à 1982, Jean Siraudeau a remis dix-huit rapports d'opération à la DAH dont dix concernent Angers et huit des localités du Maine-et-Loire. Les résultats sont exposés dans les informations archéologiques de Gallia (38-2, 1980, p. 392-399; 39-2, 1981, p. 350-357; 41-2, 1983, p. $310-312)$.

Gérard Aubin 
